Document downloaded from:

http://hdl.handle.net/10251/57592

This paper must be cited as:

Ferrando Jódar, N.; Gosalvez Ayuso, MA.; Colom Palero, RJ. (2012). Evolutionary continuous cellular automaton for the simulation of wet etching of quartz. Journal of Micromechanics and Microengineering. 22(2). doi:10.1088/0960-1317/22/2/025021

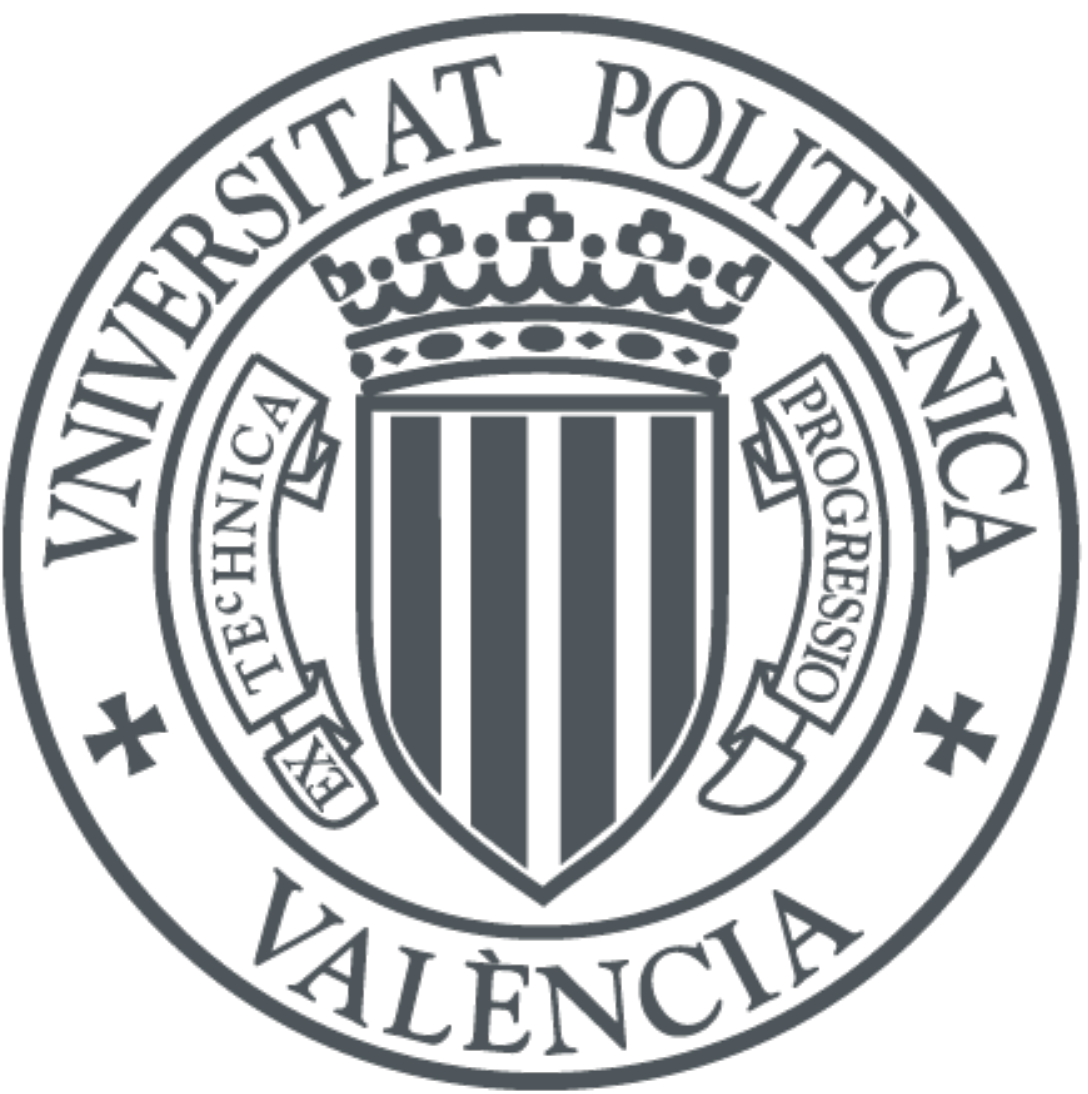

The final publication is available at

http://dx.doi.org/10.1088/0960-1317/22/2/025021

Copyright IOP Publishing: Hybrid Open Access

Additional Information 


\title{
Evolutionary Continuous Cellular Automaton for the Simulation of Wet Etching of Quartz
}

\author{
N Ferrando ${ }^{1,2}$, M A Gosálvez ${ }^{3}, \mathbf{R}$ J Colóm ${ }^{1}$ \\ ${ }^{1}$ Instituto de Instrumentación para Imagen Molecular (I3M). Centro mixto CSIC - \\ Universitat Politècnica de València - CIEMAT, camino de Vera s/n, 46022 Valencia, \\ Spain. \\ ${ }^{2}$ Centro de Física de Materiales (CFM-MPE), 20018 Donostia-San Sebastian, Spain. \\ ${ }^{3}$ Department of Materials Physics, University of the Basque Country (UPV-EHU) \\ and Donostia International Physics Center (DIPC), 20018 Donostia-San Sebastian, \\ Spain. \\ E-mail: nesferjo@upvnet.upv.es
}

\begin{abstract}
Anisotropic wet chemical etching of quartz is a bulk micromachining process for the fabrication of Micro-Electro-Mechanical Systems (MEMS), such as resonators and temperature sensors. Despite the success of the Continuous Cellular Automaton (CCA) for the simulation of wet etching of silicon, the simulation of the same process for quartz has received little attention -especially from an atomistic perspective- resulting in a lack of accurate modeling tools. This paper analyzes the crystallographic structure of the main surface orientations of quartz and proposes a novel classification of the surface atoms as well as an Evolutionary Algorithm (EA) to determine suitable values for the corresponding atomistic removal rates. Not only the presented Evolutionary Continuous Cellular Automaton (ECCA) reproduces the correct macroscopic etch rate distribution for quartz hemispheres but it is also capable of performing fast and accurate 3D simulations of MEMS structures. This is shown by several comparisons between simulated and experimental results and, in particular, by a detailed, quantitative comparison for an extensive collection of trench profiles.
\end{abstract}

PACS numbers: 89.20.Ff, 85.85.+j, 81.65.Cf

Submitted to: J. Micromech. Microeng. 


\section{Introduction}

Although silicon is the most used material for MEMS fabrication, other compounds are also popular due to their particular properties. In the case of quartz, it has outstanding piezoelectric properties, offers good electric insulation and allows optical transmission in the wavelength range of UV light. These features have favored the utilization of quartz as a substrate for the fabrication of several types of MEMS, such as resonators [1], tuning-fork probes [2] or microlenses [3].

When designing a MEMS device, the development and use of models and simulators can significantly improve the design flow, specially in complex processes where the results vary greatly depending on the initial conditions. One notable case is the modelling and simulation of the anisotropic wet etching process for the micromachining of silicon-based MEMS. In the last years the development of atomistic models based on Continuous Cellular Automata (CCA) has allowed the accurate simulation of this process for a wide range of etchants $[4,5,6,7,8]$. Despite their short history, these models have already proven their utility in practical applications $[9,10]$.

In the case of the micromachining of quartz-based MEMS, wet etching in saturated $\mathrm{NH}_{4} \mathrm{HF}_{2}$ is one of the most widely used methods, benefitting from similar features as silicon etching: low cost, the creation of flat surfaces and the possibility of batch processing. Although the complex anisotropy of the process has been analyzed in multiple studies $[11,12,13,14,15]$, only limited efforts have been made in order to obtain reliable models that properly describe the quartz etch anisotropy $[11,13]$. Based on geometric methods, the already existing models show a limited accuracy, resulting specially problematic in certain situations, such as the intersection of planes or wafer perforation.

In this paper, we present a new atomistic model for the description of anisotropic etching of quartz and an evolutionary algorithm to determine meaningful values for the parameters of the model. This enables the fast and precise simulation of quartz etching for the entire range of crystallograhic orientations. Once calibrated, the proposed model allows to simulate the micromachining of any 3-D quartz structure in just a few seconds, including two side etching with wafer perforation.

The paper is divided into three major sections. The new atomistic CCA model for quartz etching is presented first, focusing on the differences between different atom neighborhoods for the main surface orientations of quartz. Next, we describe the evolutionary algorithm that is applied to adjust the model anisotropy based on experimental etch rates as input data. Finally, we present typical results of the model convergence and several real case microstructures, comparing the results of the CCA model to the experimental structures obtained by using similar initial parameters. 


\section{Continuous Cellular Automaton for Quartz anisotropic wet etching}

\subsection{CCA for crystalline silicon}

Cellular Automata (CA) are models where space and time are described using discrete values. Typically, a CA is formed by a lattice of individual units, referred to as cells, where each cell interacts with the nearby ones. On each time step the state of each cell is defined as a function of its previous state and the state of the neighbors. The state of all cells evolves in a parallel, synchronous way. The CA modelling philosophy tries to define microscopic relationships between neighboring cells in order to describe an observed macroscopic behavior as the result of coupled cell modifications in successive time steps.

For silicon etching models that are based on the CA approach, the lattice represents the crystalline structure and the cells designate the crystallographic sites where the silicon atoms are located. In this context, the sites can have two possible states, namely, occupied by an atom (1) or empty (0), and the surface sites can be classified according to their neighborhood in terms of four indices $\left(n^{1 s}, n^{1 b}, n^{2 s}, n^{2 b}\right)$, which take into account the number of surface nearest neighbors $\left(n^{1 s}\right)$, bulk nearest neighbors $\left(n^{1 b}\right)$, surface nextnearest neighbors $\left(n^{2 s}\right)$ and bulk next-nearest neighbors $\left(n^{2 b}\right)[4,5,6]$. In a particularly successful CA method, known as the Continuous Cellular Automata (CCA), the sites are allowed to have any occupation value in the continuous range $[0,1]$, thus enabling the description of smooth parameter variations across the silicon/etchant interface and an essentially continuous propagation of the simulated surface $[6,16,17,4]$.

The etching process in the CCA method is understood as a continuous decrease of the occupation ( $\Pi$ ) of the surface sites. When a bulk atom becomes a surface atom due to the removal of one or several of its nearest neighbors, $\Pi$ is initialized with value ' 1 '. During the simulation, $\Pi$ is gradually reduced until it reaches value ' 0 ' (completely removed). At each time step ( $k$ ), the reduction in the occupation of the $i$-th surface site $\left(\Pi_{i}\right)$ is equal to the current value of the removal rate of the atom that populates the site $\left(r_{i}\right)$, multiplied by the time step $(\Delta t)$ :

$$
\Pi_{i}^{(k+1)}=\Pi_{i}^{(k)}-\Delta t \cdot r_{i}^{(k)},
$$

where the atom removal rate $r_{i}$ depends on the number of neighbors, as in:

$$
r_{i}^{(k)}=R\left(n_{i}^{1 s}(k), n_{i}^{1 b}(k), n_{i}^{2 s}(k), n_{i}^{2 b}(k)\right) .
$$

Here the particular values of $R$ depend on the actual behavior of the etchant (such as $\mathrm{KOH}, \mathrm{TMAH}$, etc...), including its concentration and temperature. Until a surface atom is completely removed, its removal rate suffers a number of changes as the numbers of surface and bulk nearest and next-nearest neighbors are modified due to the removals of nearby atoms. For this reason, $r_{i}$ and $n_{i}^{1 s}, n_{i}^{1 b}, \ldots$ are written as functions of the time step $k$ in Equations 1 and 2. Equation 1 is referred to as the principle of rate equivalence, stating that the rate of decrease of the occupation of a site is, at any moment, equal to the rate of removal of the atom populating it. In other words, the occupancy reduction rate is equal to the removal rate of the populating atom. 


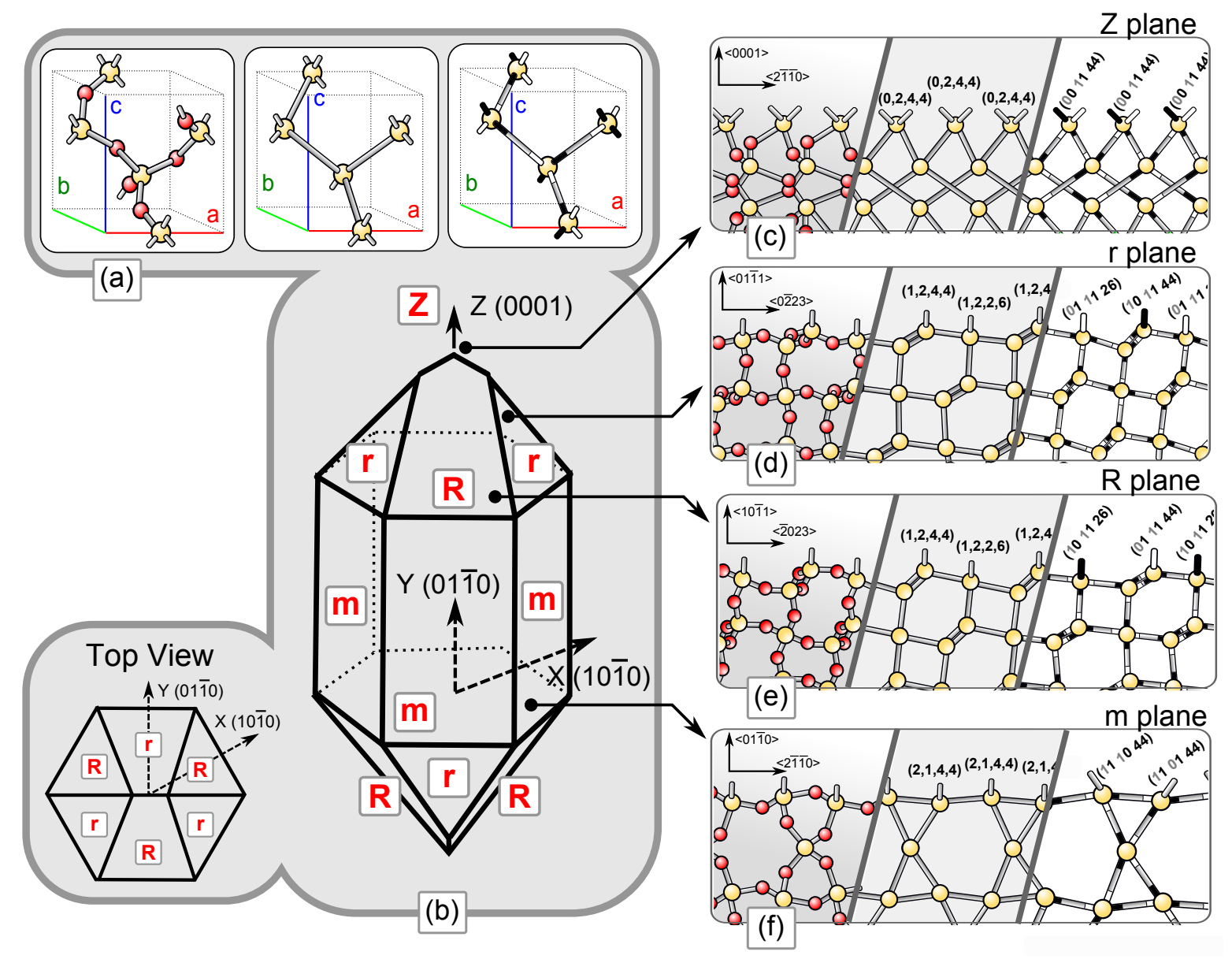

Figure 1. Overview of the quartz structure: (a) Quartz unit cell: (left) conventional, (center) simplified and (right) proposed; (b) main quartz planes during crystal growth; (c)-(f) ideal crystallographic cut for the main quartz orientations: (left) conventional structure, (center) simplified CCA model and (right) proposed CCA model.

These removal rates have been obtained traditionally by deriving and solving a set of equations that relates the etch rates of certain macroscopic planes to the sequence of occupation reductions and atom removals appearing on those planes [6]. Nevertheless, it has been demonstrated recently that the use of an Evolutionary Algorithm (EA) provides a reliable method to determine the removal rates based on macroscopic etch rates as input data [8]. The EA has made possible the calibration of the CCA for a wide range of silicon etchants, including $\mathrm{KOH}, \mathrm{KOH}+\mathrm{IPA}$, TMAH, TMAH+Triton and isotropic etching [8].

\section{2. $C C A$ for Quartz}

In order to model adequately the anisotropic etching of quartz, we adapt the previous, silicon-based CCA model to the particular crystallographic structure of the new material. The crystallographic unit cell of quartz is shown in Figure 1(a)-left. The structure is composed of silicon $(\mathrm{Si})$ and oxigen $(\mathrm{O})$ atoms, where every $\mathrm{O}$ is always 
located between two Si. Figure 1(a)-center shows a simplified unit cell that ignores the $\mathrm{O}$ atoms. Maintaining just the $\mathrm{Si}$ atoms provides enough information about the different neighborhoods for every possible surface atom, in such a manner that the etching process can be successfully described for most crystallographic planes. The exception to this rule are the $\langle 10 \overline{1} 1\rangle$ and $\langle 01 \overline{1} 1\rangle$ planes, typically referred to as $R$ and $r$, respectively, as shown in figure $1(\mathrm{~b})$. Observed from the $\mathrm{Z}$ axis, quartz anisotropic etching shows a three-fold symmetry -instead of six-fold- meaning that the $R$ and $r$ planes exhibit different etch rates. This effect has been reported in many experimental studies $[11,12]$. Taking a closer look at the atomistic structure (e.g. by comparing figures $1(\mathrm{~d})$ and (e), focusing on the left and center frames), it becomes apparent that both planes display slightly different atomistic surface sites, justifying a different behavior.

In order to assign different etch rates to the $R$ and $r$ planes, a more detailed atom classification needs to be considered. The proposed unit cell is shown in Figure 1(a)right. Although this unit cell also ignores the $\mathrm{O}$ atoms, it differentiates the first neighbors into two groups, referred to as white and black nearest neighbors, depending on their relative spatial location with respect to the silicon atom. Truly speaking, it is the four bonding directions of each atom that are defined to be black or white, as shown in Figure 1(a)-right. Each atom behaves both as a black and a white neighbor, depending on which atom it is looked from. According to this new scheme, the classification of the surface sites depends on six indices, assigned to the numbers of: nearest white surface neighbours $\left(n_{W}^{1 s}\right)$, nearest black surface neighbours $\left(n_{B}^{1 s}\right)$, nearest white bulk neighbours $\left(n_{W}^{1 b}\right)$, nearest black bulk neighbours $\left(n_{B}^{1 b}\right)$, next-nearest surface neighbours $\left(n^{2 s}\right)$, and next-nearest bulk neigbours $\left(n^{2 b}\right)$. Thus, the atomistic configurations are referred to as $\left(n_{W}^{1 s} n_{B}^{1 s} n_{W}^{1 b} n_{B}^{1 b} n^{2 s} n^{2 b}\right)$ or, equivalently, $\left(\frac{n_{W}^{1 s} n_{B}^{1 s}}{n_{W}^{1 b} n_{B}^{1 b}} n^{2 s} n^{2 b}\right)$ from now on. Using this scheme, the surface atoms appearing on planes $R$ and $r$ are labeled with different neighborhoods, as shown on the right frames of figures $1(\mathrm{~d})$-(e). This makes possible to assign different etch rates to slightly different atom types.

\subsection{Etch rate formulas for the main planes}

In the CCA method the time evolution of each surface orientation consists in the removal of specific groups of atoms in alternation [6]. In this section the particular sequence of atom removals is analyzed for the main crystallographic orientations of quartz. This provides a basic knowledge of the quartz etching process and enables the derivation of the mathematical expressions that describe the etch rate of each plane as a function of the removal rates of the corresponding surface atoms. Most importantly, the obtained formulas can be used to fix the values of several variables, thus restricting the parameter space for the evolutionary search. This speeds up the convergence of the evolutionary algorithm and ensures the correct etch rate value for the main crystallographic planes.

Figure 2 shows the relevant surface sites and the proposed atom removal sequence for the four main planes of quartz: $Z=(0001), m=(10 \overline{1} 0), r=(01 \overline{1} 1)$ and $R=(10 \overline{1} 1)$. Figure $1(\mathrm{~b})$ shows the spatial orientation of these planes in the quartz 


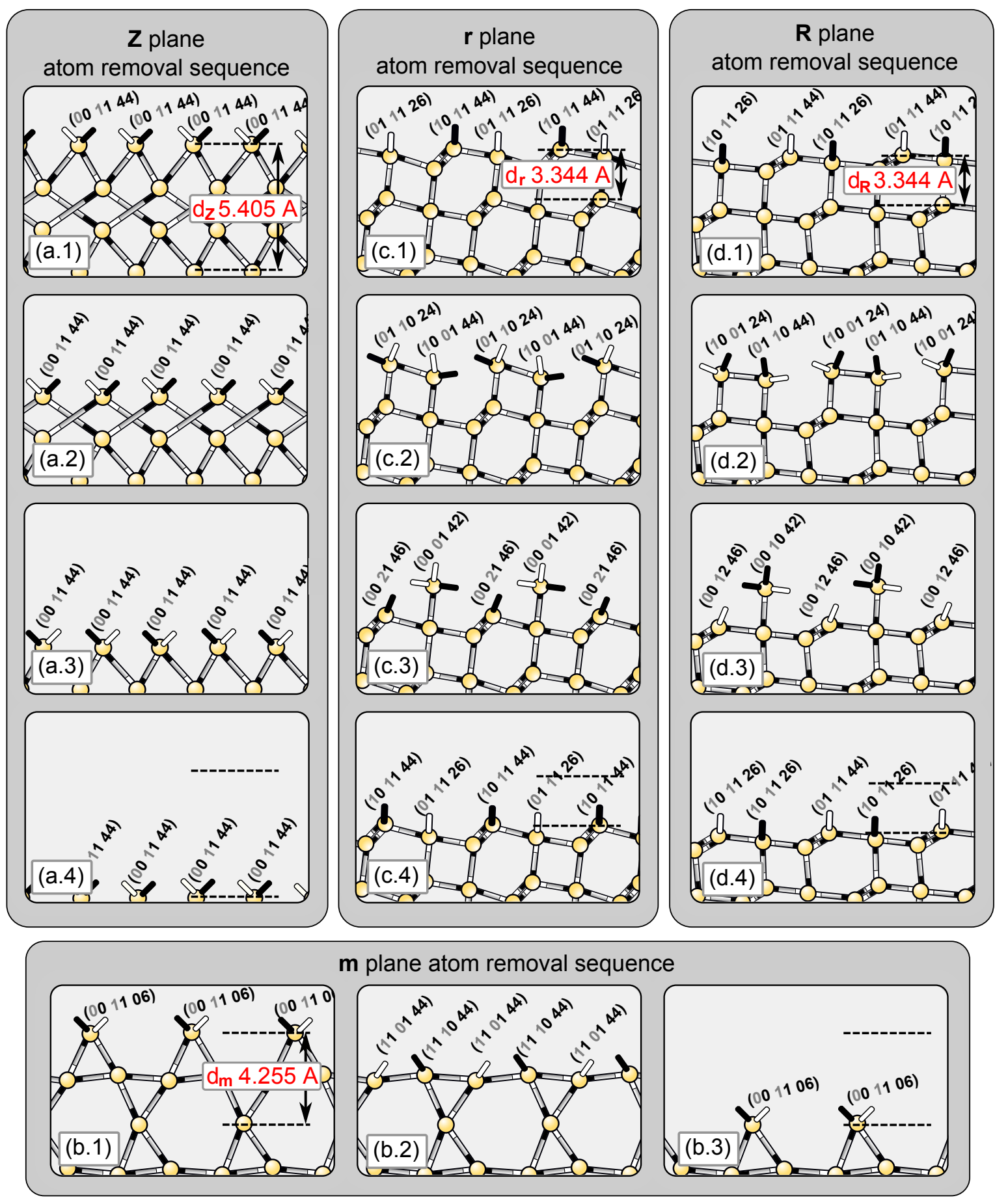

Figure 2. Proposed atom removal process during chemical wet etching of the main quartz orientations: (a) $Z$ plane, (b) $m$ plane, (c) $r$ plane, (d) $R$ plane. 
substrate. Considering any one of the planes, the derivation of the etch rate formula is based on two concepts [6]: (i) the crystallographic structure is periodic and, thus, the process can be understood as a fixed sequence of atom removals that is repeated periodically; and (ii) the removal rates of the surface atoms follow a particular hierarchy, whereby the atoms with fewer neighbors (i.e. lower coordination number) have larger removal rates. This is referred to as the step-flow or peeling mechanism for silicon [6] and quartz [14]. The steps (terraces) on the different surfaces are formed by weakly (strongly) bounded atoms and the etch front advances mostly due to the removal of the step atoms as they typically have larger removal rates.

The macroscopic etch rate $R$ is obtained as the ratio $d / t$, where $d$ is the distance advanced by the plane due to the sequence of atom removals $\left(d=\sum_{i=0}^{n} \Delta H_{i}\right)$ and $t$ is the elapsed time $\left(t=\sum_{i=0}^{n} \Delta t_{i}\right)$ :

$$
R=\frac{\sum_{i=0}^{n} \Delta H_{i}}{\sum_{i=0}^{n} \Delta t_{i}} .
$$

The distance advanced by the plane $\sum_{i=0}^{n} \Delta H_{i}$ can be obtained by analyzing the atomic structure after each atom removal, as described for the main planes in figure 2 . As an example, the atom removal sequence for the $Z$ plane consists in the consecutive removal of three atom layers, each formed by $\left(\frac{00}{11} 44\right)$ atoms (figure 2 (a)). Thus, $d_{Z}=\sum_{i=0}^{n} \Delta H_{i}=5.405 \AA$ and $t_{Z}=\sum_{i=0}^{n} \Delta t_{i}=3 t_{\left(\frac{00}{11} 44\right)}=3 / r_{\left(\frac{00}{11} 44\right)}$. Hence, we have:

$$
R_{Z}=\frac{d_{Z}}{3 t_{\left(\frac{00}{11} 44\right)}}=\frac{1}{3} r_{\left(\frac{00}{11} 44\right)} d_{Z}
$$

If $R_{Z}$ is determined experimentally, we can obtain $r_{\left(\frac{00}{11} 44\right)}$ by solving equation 4 .

The $m$ plane is characterized by having the lowest etch rate in quartz [11, 12]. Figure 2(b) shows that the surface structure of this plane is composed of two layers, one containing two types of atoms strongly bonded to the surface $\left(\left(\frac{11}{10} 44\right)\right.$ and $\left.\left(\frac{11}{01} 44\right)\right)$ and another layer containing a single atom type with a weaker bond $\left(\left(\frac{00}{11} 06\right)\right)$. In order to simplify the atom extraction sequence, we set $r_{\left(\frac{11}{10} 44\right)}$ and $r_{\left(\frac{11}{01} 44\right)}$ to be equal so that both atoms are removed simultaneously. As a result, the atoms $\left(\frac{11}{10} 44\right)$ and $\left(\frac{11}{01} 44\right)$ are removed in the time increment $t_{\left(\frac{11}{10} 44\right)}$ while the atoms $\left(\frac{00}{11} 06\right)$ are removed in the time increment $t_{\left(\frac{00}{11} 06\right)}$. This resembles the removal sequence for the (111) silicon surface [6]. Thus, the equation for the macroscopic etch rate becomes:

$$
R_{m}=\frac{d_{m}}{t_{\left(\frac{11}{10} 44\right)}+t_{\left(\frac{00}{11} 06\right)}}=\frac{d_{m} r_{\left(\frac{11}{10} 44\right)} r_{\left(\frac{00}{11} 06\right)}}{r_{\left(\frac{11}{10} 44\right)}+r_{\left(\frac{00}{11} 06\right)}},
$$

where $d_{m}=4.255 \AA$ is the advanced distance after removing both atom layers. Due to the extremely low reactivity of this plane and the strong bonding of $\left(\frac{11}{10} 44\right)$ and $\left(\frac{11}{01} 44\right)$, our approach for solving this equation is to fix the etch rates of $r_{\left(\frac{11}{10} 44\right)}=r_{\left(\frac{11}{01} 44\right)}$ to a 
sufficiently small value $\left(\approx 1 \cdot 10^{-4}\right)$ and solve equation 5 to obtain $r_{\left(\frac{00}{11} 06\right)}$ in terms of the experimental value for $R_{m}$.

The atom extraction sequence for planes $r$ and $R$ is more complicated. Figures $2(\mathrm{c})-(\mathrm{d})$ show that there are three types of surface atoms and each of them goes through two different atomistic configurations before complete removal. The proposed removal sequence extracts first the atoms with fewer neighbors (step-flow hierarchy). For example, the sequence suggested in figure 2(d) occurs when $r_{\left(\frac{01}{11} 44\right)}>r_{\left(\frac{10}{11} 26\right)}$, $r_{\left(\frac{10}{01} 24\right)}>r_{\left(\frac{01}{10} 44\right)}$ and $r_{\left(\frac{00}{10} 42\right)}>r_{\left(\frac{00}{12} 46\right)}$. Analyzing the atom removal sequence for plane $R$, the following three equations are obtained:

$$
\begin{aligned}
& r_{\left(\frac{10}{11} 26\right)} t_{\left(\frac{01}{11} 44\right)}+r_{\left(\frac{10}{01} 24\right)} t_{\left(\frac{10}{01} 24\right)}=1 \\
& r_{\left(\frac{01}{10} 44\right)} t_{\left(\frac{10}{01} 24\right)}+r_{\left(\frac{00}{10} 42\right)} t_{\left(\frac{00}{10} 42\right)}=1 \\
& r_{\left(\frac{00}{12} 46\right)} t_{\left(\frac{00}{10} 42\right)}+r_{\left(\frac{01}{11} 44\right)} t_{\left(\frac{01}{11} 44\right)}=1
\end{aligned}
$$

where $t_{\left(\frac{01}{11} 44\right)}, t_{\left(\frac{10}{01} 24\right)}$ and $t_{\left(\frac{00}{10} 42\right)}$ are the elapsed times corresponding to the atom removals performed in each step of the sequence. These equations are based on the fact that the accumulated reduction in the occupation of each surface site must be equal to 1 [6]. Solving this equation system, we obtain:

$$
\begin{aligned}
R_{R} & =\frac{d_{R}}{t_{\left(\frac{01}{11} 44\right)}+t_{\left(\frac{10}{01} 24\right)}+t_{\left(\frac{00}{10} 42\right)}} \\
& =\frac{d_{R}(A+B)}{C+D+E} ; \text { where } \\
A & =r_{\left(\frac{00}{10} 42\right)} r_{\left(\frac{01}{11} 44\right)} r_{\left(\frac{10}{01} 24\right)} \\
B & =r_{\left(\frac{00}{12} 46\right)} r_{\left(\frac{01}{10} 44\right)} r_{\left(\frac{10}{11} 26\right)} \\
C & =\left(r_{\left(\frac{00}{12} 46\right)}-r_{\left(\frac{01}{11} 44\right)}\right)\left(r_{\left(\frac{01}{10} 44\right)}-r_{\left(\frac{10}{01} 24\right)}\right) \\
D & =r_{\left(\frac{00}{10} 42\right)}\left(r_{\left(\frac{01}{11} 44\right)}+r_{\left(\frac{10}{01} 24\right)}-r_{\left(\frac{10}{11} 26\right)}\right) \\
E & =r_{\left(\frac{10}{11} 26\right)}\left(r_{\left(\frac{00}{12} 46\right)}+r_{\left(\frac{01}{10} 44\right)}\right) .
\end{aligned}
$$

Here, $d_{R}=3.344 \AA$ is the total advanced distance after removing the three types of surface atoms.

The derivation of the etch rate for the $r$ plane leads to a similar equation, but with the corresponding surface sites for this plane. For the $R$ and $r$ planes, up to six different surface sites appear, thus enabling many solutions for both equations (one for each possible sequence of removals according to the chosen hierarchy of removal rates). In our experience, appropriate results are obtained when conforming to the following restrictions ( $R$ plane):

$$
\begin{array}{ll}
r_{\left(\frac{00}{12} 46\right)} & =1 \cdot 10^{-5} \\
r_{\left(\frac{00}{10} 42\right)} & =1.0 \\
\left(r_{\left(\frac{01}{11} 44\right)}, r_{\left(\frac{10}{11} 26\right)}\right) & \ll\left(r_{\left(\frac{10}{01} 24\right)}, r_{\left(\frac{01}{10} 44\right)}\right) .
\end{array}
$$




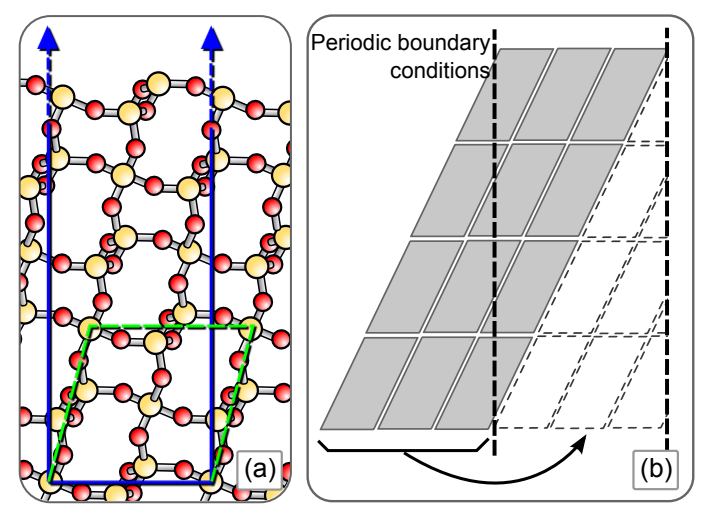

Figure 3. (a) Orthorombic and triclinic unit cell search for the $r$ plane. (b) Atom displacements to describe an orthorhombic substrate using triclinic unit cells.

The obtained expression for the $R$ plane shows that modelling the etching process at the atomic scale for quartz is significantly more complicated than for silicon, leading to complex equations even for low index planes.

\subsection{Triclinic Unit Cell}

One of the main procedures to implement the CCA for silicon etching is the creation of an orthorhombic unit cell [18]. Crystal surfaces of any orientation can be created by replicating the orthorhombic unit cell along the three axes. The use of the unit cell in combination with an octree data structure enables a significant reduction in memory use and a dramatic increase in the simulation speed for these models [19].

Due to the hexagonal crystallographic structure of quartz, finding an orthorhombic unit cell is specially difficult, resulting in huge unit cells with thousands of atoms, even for low index planes. As a practical example, obtaining an orthorhombic unit cell for a low-index plane such as $r$ can take up to 816 atoms while triclinic unit cells can be found with just 12 silicon atoms, as shown in figure 3(a). This problem is even more evident for higher index planes. In such cases, the use of an orthorhombic unit cell severely limits the ability to effectively partition the space, leading to excessive memory consumption and long simulation times.

The use of triclinic unit cells as the base for constructing the substrate has one drawback: the final shape of the substrate will be trigonal, instead of orthorhombic. Taking advantage of the periodic boundary conditions defined in the CCA [18], an orthorhombic substrate can be easily represented by artificially displacing the atoms that fall outside the limits of the theoretical orthorhombic region, as schematically shown in figure 3(b). The displacement is just performed when representing the atoms on the computer screen, leaving the underlying model intact. The use of this procedure allows simulating an orthorhombic substrate by using any type of unit cell, thus enabling the efficient simulation of any crystallographic structure. 


\section{Calibrating the CCA using an Evolutionary Algorithm}

Originally, the calibration of the CCA was based on performing a complete surface site classification and deriving a set of equations that established the relationship between the removal rates of those atoms and the etch rates at the macroscopic scale [6]. Only recently, it has been demonstrated that the use of Evolutionary Algorithms can perform this task automatically, requiring only an atom classification/equation derivation for the main cristallographic planes [8].

Compared to silicon, the identification of the surface sites for quartz is more complicated due to two reasons: (i) the occurrence of four main crystallographic orientations instead of three $(\langle 10 \overline{1} 0\rangle,\langle 0001\rangle,\langle 01 \overline{1} 1\rangle$ and $\langle 10 \overline{1} 1\rangle$, in comparison to $\langle 100\rangle$, $\langle 110\rangle$ and $\langle 111\rangle$ ) leads to a broader collection of vicinal surfaces, characterized by a wider range of surface sites and a more complex overall etch rate distribution. (ii) A larger complexity of the atom removal steps for $\langle 01 \overline{1} 1\rangle$ and $\langle 10 \overline{1} 1\rangle$ planes -where six atom types appear and three removal steps need to be performed-complicates the equation derivation procedure even for low index planes. In silicon, the etching of the main planes is simpler, consisting on the successive removal of layers of the same type of atoms. This complexity and the good results obtained in [8] for silicon etching justify the use of an evolutionary algorithm instead of manually deriving the equations for a large number of different surface orientations.

\subsection{Introduction to Evolutionary Algorithms}

An Evolutionary Algorithm (EA) is an optimization procedure based on the principles of natural selection. The EA considers a set individuals, each corresponding to a possible solution of the problem. The collection of individuals is referred to as the population. For a number of generations, the population goes through several procedures that simulate the effect of selective pressure from the environment. Only the most capable individuals survive, spreading out their characteristics in the new generations. This evolution process is usually composed of the following steps: (i) Characterization of the fitness of each individual to the environmental demands, which is implemented by evaluating an objective function over each individual, thus providing a ranking of all the individuals. (ii) Selection of a subset of individuals, in such a way that the most capable ones have a larger probability of being selected. (iii) Creation of new individuals (offspring) from the recombination of the previously selected individuals. (iv) Application of mutations to the offspring in order to explore new characteristics that may improve the performance of the offspring. (v) Reinsertion of the best offspring subset into the population.

In the spirit of the Evolvable Cellular Automata (ECA) introduced by Mitchell et al. [20], Gosalvez et al. have recently demonstrated that the evolutionary approach can be used to calibrate the CCA model parameters (removal rates of the surface atoms) in order to simulate anisotropic wet chemical etching of silicon accurately [8]. The resulting Evolutionary CCA (ECCA) properly describes any of the most used chemical 
etchants for silicon, including TMAH+Triton or isotropic etchants. For quartz etching, we focus on modifying the EA presented in [8] in order to calibrate the removal rates of the quartz surface atoms defined in section 2.2. The EA allows to obtain the removal rates of all the surface atoms that cannot be achieved by a mathematical derivation of the macroscopic etch rate equations for arbitrary surface orientations due to their complexity.

\subsection{Main Characteristics}

Due to the good results obtained for the modeling of silicon etching [8], a fair amount of design decisions regarding the EA remain similar in the current study. The main characteristics of the EA for quartz are as follows:

- The population contains $M=200$ individuals, where each individual stores a total of $8\left(n^{1 s}\right) \cdot 8\left(n^{1 b}\right) \cdot 12\left(n^{2 s}\right) \cdot 12\left(n^{2 b}\right)=9216$ etch rates, one for each theorically possible neighborhood.

- All the etch rates of the population are initialized to $R_{\left(\frac{00}{14} 4\right)} / 2+r n d$, where $r n d$ is an uniform random number in the range $[-0.125,0.125]$. If a value is reduced below $1 \cdot 10^{-4}$, it is clamped at this point. Setting an initial population with random values ensures diversity and speeds up the convergence process.

- Rank-based linear fitness assignment, with a selective pressure of 1.5.

- Roulette wheel selection for the creation of a total of 24 new individuals.

- Intermediate real-valued recombination.

- Stochastic real-valued mutation of $5 \%$ of the etch rates of each individual, using the mutation operator of the breeder genetic algorithm [21].

- Elitist reinsertion, where the best 10 offspring replace the worst 10 individuals of the population.

In order to increase the convergence speed of the overall algorithm and ensure an exact etching behavior of the main planes, the removal rates of the surface atoms appearing at planes $Z, m, R$ and $r$ are calculated analytically by using equations 4, 5 and 9 . As a practical example, when using saturated $\mathrm{NH}_{4} \mathrm{HF}_{2}$ at $70^{\circ} \mathrm{C}$ as the etchant, the etch rates for these planes are: $\left\{R_{Z}, R_{m}, R_{R}, R_{r}\right\}=$ $\{0.571,0.0138,0.2119,0.0593\} \mathrm{um} / \mathrm{min}=\{0.0951,0.0023,0.0353,0.0098\} \AA \mathrm{A} / \mathrm{ms}[11]$. From equation 4 we obtain $r_{\left(\frac{00}{11} 44\right)}=0.0528$. From equation 5 and defining $t_{\left(\frac{11}{10} 44\right)}=$ $2 \cdot t_{\left(\frac{00}{11} 06\right)}$, we obtain $r_{\left(\frac{11}{10} 44\right)}=r_{\left(\frac{11}{01} 44\right)}=5.405 \cdot 10^{-4}$ and $r_{\left(\frac{00}{11} 06\right)}=0.0016$. For equation

9, we follow the recommendations given in section 2.3 and obtain: $r_{\left(\frac{00}{10} 42\right)}=1.0$, $r_{\left(\frac{10}{01} 24\right)}=0.0697, r_{\left(\frac{01}{10} 44\right)}=0.0522, r_{\left(\frac{01}{11} 44\right)}=0.0121, r_{\left(\frac{10}{11} 26\right)}=0.0023, r_{\left(\frac{01}{12} 46\right)}=1 \cdot 10^{-5}$. For the $r$ plane, the removal rates are: $r_{\left(\frac{00}{01} 42\right)}=1.0, r_{\left(\frac{01}{10} 24\right)}=0.0195, r_{\left(\frac{10}{01} 44\right)}=0.0146$, $r_{\left(\frac{10}{11} 44\right)}=0.00341, r_{\left(\frac{01}{11} 26\right)}=6.625 \cdot 10^{-4}, r_{\left(\frac{00}{21} 46\right)}=1 \cdot 10^{-5}$.

\subsection{Objective Function}

The Objective Function (OF) is used to determine the goodness of each solution. The OF values are used to sort the individuals for selection in the recombination and 
reinsertion steps. The proposed OF for quartz keeps some similarities with the one for silicon, but it incorporates several important changes, as described below. The OF for individual $i$ is the combination of four values:

$$
\epsilon_{i}=\sum_{\alpha=1}^{4} c_{\alpha} \epsilon_{\alpha, i}, \quad i \in\{1,2, \ldots, M\},
$$

where each $\epsilon_{\alpha, i}$ with $\alpha \in\{1,2,3,4\}$ quantifies a different type of error found for individual $i$ and the four parameters $c_{\alpha}$ with $\alpha \in\{1,2,3,4\}$ are used to determine a weighted average of the four errors. The set $\left[c_{1}, c_{2}, c_{3}, c_{4}\right]=[0.7,0.25,0.035,0.015]$ gives a balanced relevance to all parameters, resulting in good convergence to the optimal solution. In order to mix contributions of totally different nature, the $\epsilon_{\alpha, i}$ errors are population-normalized:

$$
\epsilon_{\alpha, i}=E_{\alpha, i} / E_{\alpha}^{\min }, \quad i \in\{1,2, \ldots, M\},
$$

where $E_{\alpha, i}$ represents the raw version of the calculated error and $E_{\alpha}^{\text {min }}=$ $\min \left\{E_{\alpha, i}\right\}_{i=1,2, \ldots, N}$ represents the minimum (best) value of $E_{\alpha, i}$ found for the whole population. Centering our attention on one individual $i$ at random, we simplify the notation by dropping the index $i$ for the rest of this section and focus on the presentation of the four types of error, namely, $E_{1}$ through $E_{4}$.

3.3.1. $E_{1}$ : Hemisphere anisotropy error. Due to exhibiting all possible crystallographic orientations of the substrate, the etching of a convex hemisphere has been traditionally used as a successful method to obtain information of the etch rate distribution of various etchants for both quartz and silicon [11,22]. The calculation of $E_{1}$ is based on the comparison of the corresponding experimental and simulated etch rate distributions. Smaller values of $E_{1}$ mean that the simulated etch rate distribution by the CCA is closer to the experimental one.

The experimental and simulated hemispheres are sampled before and after etching, obtaining the radial advancement of the etch front at multiple locations, in particular every $2^{\circ}$ of latitude $(\theta)$ and longitude $(\phi)$ in the ranges of $0 \leq \theta \leq 90^{\circ}$ and $0 \leq \phi \leq 360^{\circ}$. The etch rate for each location is calculated by dividing the radial displacement by the etch time. Figures 4(a)-(c) show the pre-etch and post-etch spheres as well as a stereographic projection of the resulting etch rate distribution for the CCA model. Figure 4(d) shows the etch rate distribution obtained by the etching of a quartz hemisphere with saturated $\mathrm{NH}_{4} \mathrm{HF}_{2}$ [11].

The mathematical expression for this comparison is:

$$
E_{1}=\frac{1}{45 \cdot 180}\left(\sum_{\theta=0}^{90^{\circ}(2)} \sum_{\phi=0}^{360^{\circ}(2)} \frac{R_{\theta, \phi}^{e x p, *}-R_{\theta, \phi}^{s i m}}{R_{\theta, \phi}^{e x p, *}}\right),
$$

where $R^{e x p, *}$ is an averaged value of the experimental etch rate, which takes into account the three-fold symmetry of the etch rate distribution for quartz: $R_{\theta, \phi}^{e x p, *}=$ $\left(R_{\theta, \phi}^{\exp }+R_{\theta, \phi+120}^{\exp }+R_{\theta, \phi+240}^{\exp }\right) / 3$. Unlike silicon, some crystallographic orientations of quartz only appear in the equator, such as the $m$ planes in the case of a $\langle 0001\rangle$-oriented 


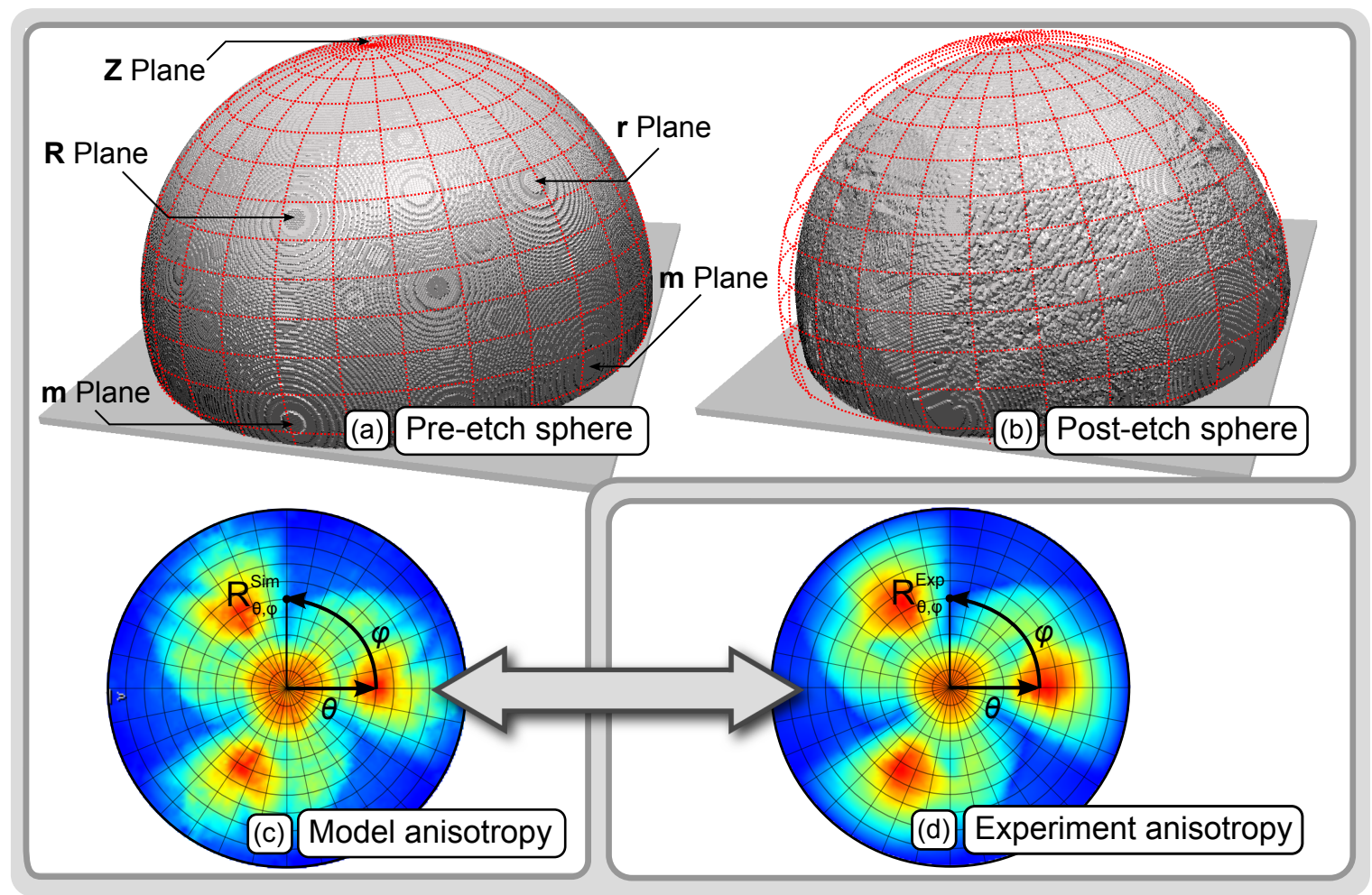

Figure 4. (a)-(c) Simulated etching: (a) pre-etch hemisphere, (b) post-etch hemisphere, and (c) stereographic projection of the obtained etch rate distribution for $\mathrm{NH}_{4} \mathrm{HF}_{2}$. (d) Corresponding experimental etch rate plot.

quartz-hemisphere. To avoid any artifacts in the equator etch rates, the experimental and simulated quartz hemispheres span a larger latitude range $\left(0<\theta<100^{\circ}\right)$ than theoretically needed $\left(0<\theta<90^{\circ}\right)$.

Note that one etching simulation must be performed per population individual in order to determine $E_{1}$ and that the EA generates 24 new individuals per iteration while it can take up to $2-4 \cdot 10^{3}$ iterations for the EA to converge, thus leading to several tens of thousands of simulations. To make this large number of simulations feasible, an efficient CCA simulator is needed. We use a fast, GPU-based implementation [19], whereby each hemisphere simulation takes about 3 seconds. The initial hemisphere surface contains 472298 surface atoms and $\sim 1.4 \cdot 10^{6}$ atoms are removed per simulation. The total computing time for $E_{1}$ is approximately 60 hours for 3000 iterations.

\subsection{2. $E_{2}$ : Underetching, 3-fold symmetry and floor corrugation. Although the etching} of a sphere allows to obtain the etch rates for a wide range of orientations, several artifacts may appear when simulating the etching of a MEMS structure, such as an incorrect symmetry in the etched structure, disproportionate underetching at convex corners and/or excessive floor corrugation, all of which are features not obviated by the etching of the hemisphere [8]. To avoid such artifacts, we monitor the etching of a masked (0001) quartz chip, focusing on promoting three-fold symmetry, minimum 

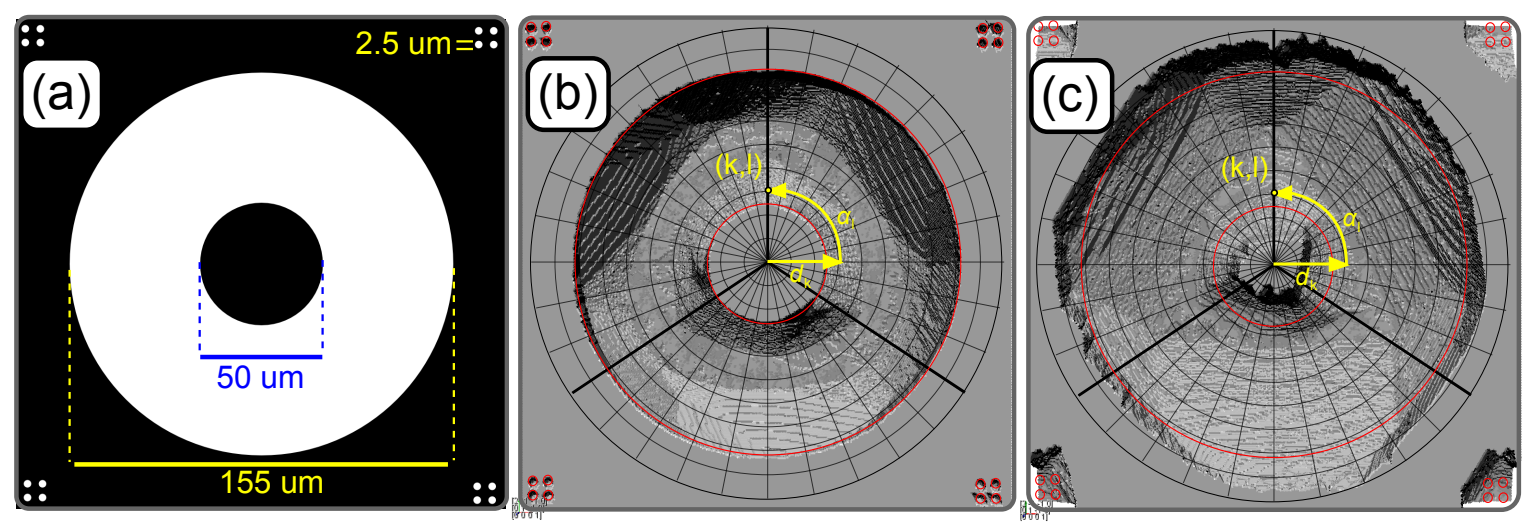

Figure 5. (a) Mask used to determine $E_{2}$. (b) Good calibration (low $E_{2}$ ). (c) Bad calibration (high $E_{2}$ ).

underetching and minimum floor corrugation, as observed in experiments [13]. We use a chip size of $128 \times 128 \mu \mathrm{m}^{2}$ and a $\mathrm{Cr} / \mathrm{Au}$ mask pattern containing a central unmasked ring (52.5 $\mu \mathrm{m}$ wide) and a set of small unmasked circles $(2.5 \mu \mathrm{m}$ wide $)$ located in the periphery of the mask, as shown in figure 5(a). Wet etching is simulated to a depth of $25 \mu \mathrm{m}$.

After the etching simulation, the mask is removed and the etch depth is sampled all over the chip, resulting in a 2D matrix of 200x200 equally-spaced, depth measurements. We refer to this matrix by using $(i, j)$ cartesian coordinates, where $0<i<I-1$ and $0<j<J-1$, in steps of 1 and using $I=J=200$. Alternatively, we define a 2D spiderweb matrix of 380x360 depth locations, as sketched in figure 5(b). We refer to this matrix by using $(k, l)$ polar coordinates, where $0<k<K-1$ in steps of 0.25 and $K=95$, and $0<l<L-1$ in steps of 1 and $L=360$. The index $k$ indicates the distance to the center of the spiderweb $\left(d_{k}\right)$, with $k=0$ denoting the center and $k=K-1$ denoting the largest circumference. The index $l$ indicates the value of angle $\alpha_{l}$. The two matrices are then used to characterize the following four components of $E_{2}$ :

- Differences in the 3-fold symmetry on the etched central ring. According to the quartz anisotropy, points at the same distance from the center and separated $120^{\circ}$ should have the same etch depth. Mathematically, we focus on minimizing:

$$
E_{2}^{A U}=a_{1} \sum_{k=0}^{K-1(0.25)} \sum_{l=0}^{L}\left(\left|H_{(k, l)}-H_{(k, l+120)}\right|\right) .
$$

- Underetching at the borders of the main ring. The inner $\left(k=K_{1}=23.5\right)$ and outer $\left(k=K_{2}=74\right)$ ring edges are monitored, accumulating the etch depth at these locations. Technically, we focus on minimizing the following measure:

$$
E_{2}^{U E, I}=a_{2} \sum_{l=0}^{L}\left(\sum_{k=0}^{K_{1}(0.25)}\left|H_{(k, l)}\right|+\sum_{k=K^{2}}^{K-1(0.25)}\left|H_{(k, l)}\right|\right) .
$$

- Underetching at the unmasked periphery circles. The small circles should lead to the early development of slow etching planes, resulting in a very small amount of 
removed material. Thus, we choose to monitor the amount of removed material around the circles by accumulating the etched depth in these regions and focus on minimizing it:

$$
E_{2}^{U E, I I}=a_{3} \sum_{i=0}^{25} \sum_{j=0}^{25}\left(\left|H_{(i, j)}\right|+\left|H_{(i+175, j)}\right|+\left|H_{(i, j+175)}\right|+\left|H_{(i+175, j+175)}\right|\right) \cdot(16
$$

- We focus on minimizing the difference between the theoretical etch rate of the $\langle 0001\rangle$ plane and the time needed for reaching the desired depth:

$$
E_{2}^{F C}=1+a_{4}\left(H_{\max } / H_{\text {calc }}-1\right)
$$

where $H_{\max }=\max \left\{H_{i, j}\right\}=\max \left\{H_{k, l}\right\}$ is the maximum depth obtained in the simulation and $H_{\text {calc }}=\frac{1}{3} r_{\left(\frac{00}{11} 4\right)} d_{Z} \cdot \Delta t$ is the theoretical depth, calculated by using the simulation time $\Delta t$, the removal rate $r_{\left(\frac{00}{11} 4\right)}$ for the current population individual and $d_{Z}=5.405 \AA . \quad E_{2}^{F C}=1$ when the theoretical and simulated values coincide while $E_{2}^{F C}$ grows when the two measures differ.

Once $E_{2}^{A U}, E_{2}^{U E, I}, E_{2}^{U E, I I}$ and $E_{2}^{F C}$ have been determined, $E_{2}$ is calculated as follows:

$$
E_{2}=\left(E_{2}^{A U}+E_{2}^{U E, I}+E_{2}^{U E, I I}\right) \cdot E_{2}^{F C}
$$

Inspection of equations 14-17 shows that each component has a coefficient $a_{i}$ that controls the weight in the final equation. The set $\left[a_{1}, a_{2}, a_{3}, a_{4}\right]=[0.2,0.011,0.1,4]$ has been tested to give a suitable balance between all components. Figures 5(b)(c) compare the results associated to a small and large value of $E_{2}$, respectively. In particular, figure 5(c) corresponds to the best individual of an early population (far from convergence), featuring most artifacts described in this section, specially regarding excessive underetching.

Regarding the computational cost, the chip surface is composed of 88101 atoms and about $1.2 \cdot 10^{6}$ atoms are removed in the process, taking about 2 sec per simulation by using our GPU implementation. Since $E_{2}$ must be determined for each of the 24 new individuals generated in each iteration, the total required computational time for this coefficient is 40 hours for 3000 generations.

3.3.3. $E_{3}$ : Step flow hierarchy error. Following a similar approach as in Ref. [8], E $E_{3}$ tries to promote the effect of step-flow or peeling. This is done by monitoring the ratio $\frac{r_{A}}{r_{B}}$ for various pairs of surface atoms where type $A$ is expected to have a lower removal rate than type $B\left(\frac{r_{A}}{r_{B}}<1\right)$. Thus, we focus on minimizing the following measure:

$$
\begin{aligned}
& E_{3}=\sum_{n_{W}^{1 s}=0}^{3} \sum_{n_{B}^{1 s}=0}^{\left(3-n_{W}^{1 s}\right)} \sum_{n_{W}^{1 b}=0}^{\left(3-n_{W}^{1 s}-n_{B}^{1 s}\right)} \sum_{n_{B}^{1 b}=0}^{\left(3-n_{W}^{1 s}-n_{B}^{1 s}-n_{W}^{1 b}\right)}
\end{aligned}
$$

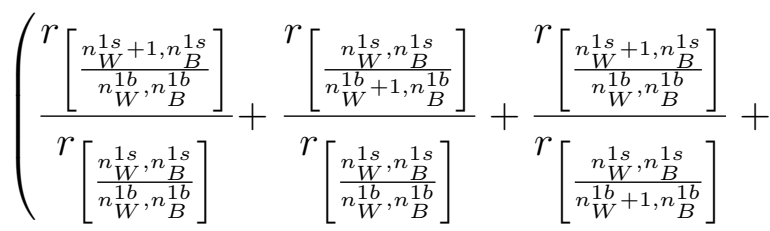




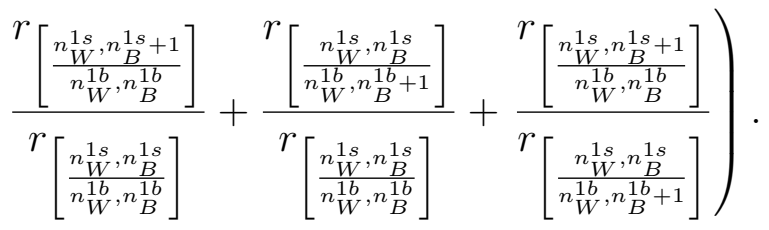

Each removal rate $r$ shown in equation 19 is obtained by averaging all the removal rates of the surface atom types that share the same $\left(\frac{n_{W}^{1 s}, n_{B}^{1 s}+1}{n_{W}^{1 b}, n_{B}^{1 b}}\right)$ neighborhood. The minimization of $E_{3}$ encourages lowering the average etch rate of the surface atoms with a more populated first neighborhood. As described in Ref. [8], the surface sites that do not appear in the simulations are not taken into account as they are not optimized by the EA.

3.3.4. $E_{4}$ : Removal rate spread error. $E_{4}$ tries to prevent an excessive spread of the etch rates with similar first neighborhood. This prevents disproportionately large or small removal rates for particular surface sites that can differ in value significantly from the removal rates of similar surface atoms. For example, a slight modification in the second neighborhood should not lead to a drastic change in the removal rate. The chosen expression for $E_{4}$ is:

$$
E_{4}=\sum_{n_{W}^{1 s}=0}^{3} \sum_{n_{B}^{1 s}=0}^{\left(3-n_{W}^{1 s}\right)} \sum_{n_{W}^{1 b}=0}^{\left(3-n_{W}^{1 s}-n_{B}^{1 s}\right)} \sum_{n_{B}^{1 b}=0}^{\left(3-n_{W}^{1 s}-n_{B}^{1 s}-n_{W}^{1 b}\right)} \sigma_{\left[\begin{array}{l}
n_{W}^{1 s}, n_{B}^{1 s} \\
n_{W}^{1 b}, n_{B}^{1 b}
\end{array}\right]}^{2}
$$

where $\left.\sigma_{\left[\frac{n_{W}^{1 s}, n_{B}^{1 s}}{n_{W}^{1 b}, n_{B}^{16}}\right.}^{2}\right]$ is the population variance of all the surface sites that share the

same $\left(\frac{n_{W}^{1 s}, n_{B}^{1 s}}{n_{W}^{1 b}, n_{B}^{16}}\right)$ first neighborhood, as described in Ref. [8]. As for $E_{3}$, the surface sites that do not appear in the simulations are disregarded as they are not optimized by the EA.

\section{Results}

The proposed CCA model and modified EA algorithm have been implemented as a new module for the already existing atomistic wet etching simulator (IntelliEtch [23]). The test to verify the performance of the overall proposed method consists on the calibration of three experimentally characterized etchants: $\mathrm{NH}_{4} \mathrm{HF}_{2}$ at $70^{\circ} \mathrm{C}[11], 80$ ${ }^{\circ} \mathrm{C}[11]$ and $85^{\circ} \mathrm{C}[15]$. The computing platform consists of a Intel Core 2 Duo E4500 with 2 GB of RAM and a Nvidia GeForce GTX260. Having a similar etch rate anisotropy but very different reactivity in absolute terms, the three etchant calibrations lead to a similar, satisfactory convergence process. Here we present the convergence details for $\mathrm{NH}_{4} \mathrm{HF}_{2}$ at $70^{\circ} \mathrm{C}$. The accuracy of the obtained calibrations is tested by comparing several CCA simulations of various microstructures with the experimental results using $\mathrm{NH}_{4} \mathrm{HF}_{2} 85^{\circ} \mathrm{C}$. 


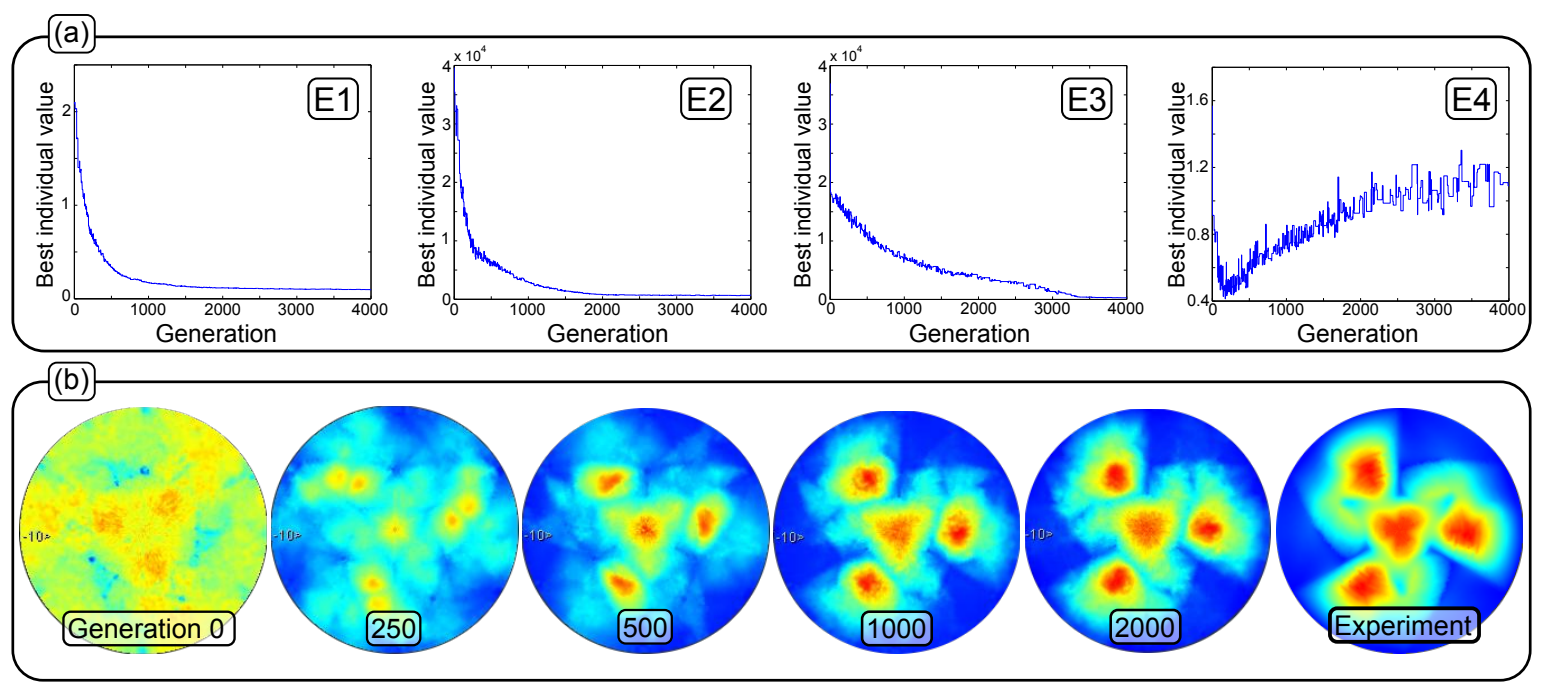

Figure 6. Evolutionary calibration of $\mathrm{NH}_{4} \mathrm{HF}_{2} 70^{\circ} \mathrm{C}$ : (a) Evolution of the $E_{1} \ldots E_{4}$ error measures. (b) Evolution of the etch rate distribution for the best population individual for the etching of the hemispherical sample.

\subsection{CCA calibration: EA convergence}

The EA takes usually a few thousands of generations in order to reach an optimal solution. The result is considered to be converged when the coefficients $E_{1}$ through $E_{4}$ become stabilized and their final value is close to 0 . Figure $6\left(\right.$ a) shows the values for $E_{1}$ through $E_{4}$ for the best individual along 4000 iterations for the calibration of $\mathrm{NH}_{4} \mathrm{HF}_{2}$ at $70^{\circ} \mathrm{C}$. As can be seen, $E_{1}$ and $E_{2}$ are reduced quickly in the first 1000 generations. Having a lower weight, $E_{3}$ converges slowly. Reducing $E_{3}$ usually leads to less noisy simulations and, thus, waiting for its convergence is recommended. Having the lowest weight, $E_{4}$ essentially maintains a converged value after the first 2000 generations. The amount of surface sites detected and optimized by the EA is $\approx 900$ in all cases.

Figure 6 (b) shows the stereographic projection of the etch rate of the best individual when etching a quartz hemisphere at several representative generations $(0,250,500$, 1000 and 2000). After 2000 generations, the CCA model is already able to describe the etchant anisotropy with good accuracy. To check for repeatability, similar evolutionary calibrations have been carried out for the three specified etchants, obtaining that the best individual presents acceptably good characteristics in all the cases after 2000-3000 iterations.

\subsection{CCA simulations: Comparison with real case structures}

In this section the accuracy of the CCA model calibrated with the proposed evolutionary algorithm is analyzed. The selected etchant is saturated $\mathrm{NH}_{4} \mathrm{HF}_{2}$ at $85^{\circ} \mathrm{C}$. The etch rate distribution for this etchant has been obtained by modifying the etch rate data of the same etchant at $80^{\circ} \mathrm{C}$ [11], based on the overall reactivity and anisotropy of the main planes at $85^{\circ} \mathrm{C}$, as extracted from Ref. [15]. After the evolutionary calibration 


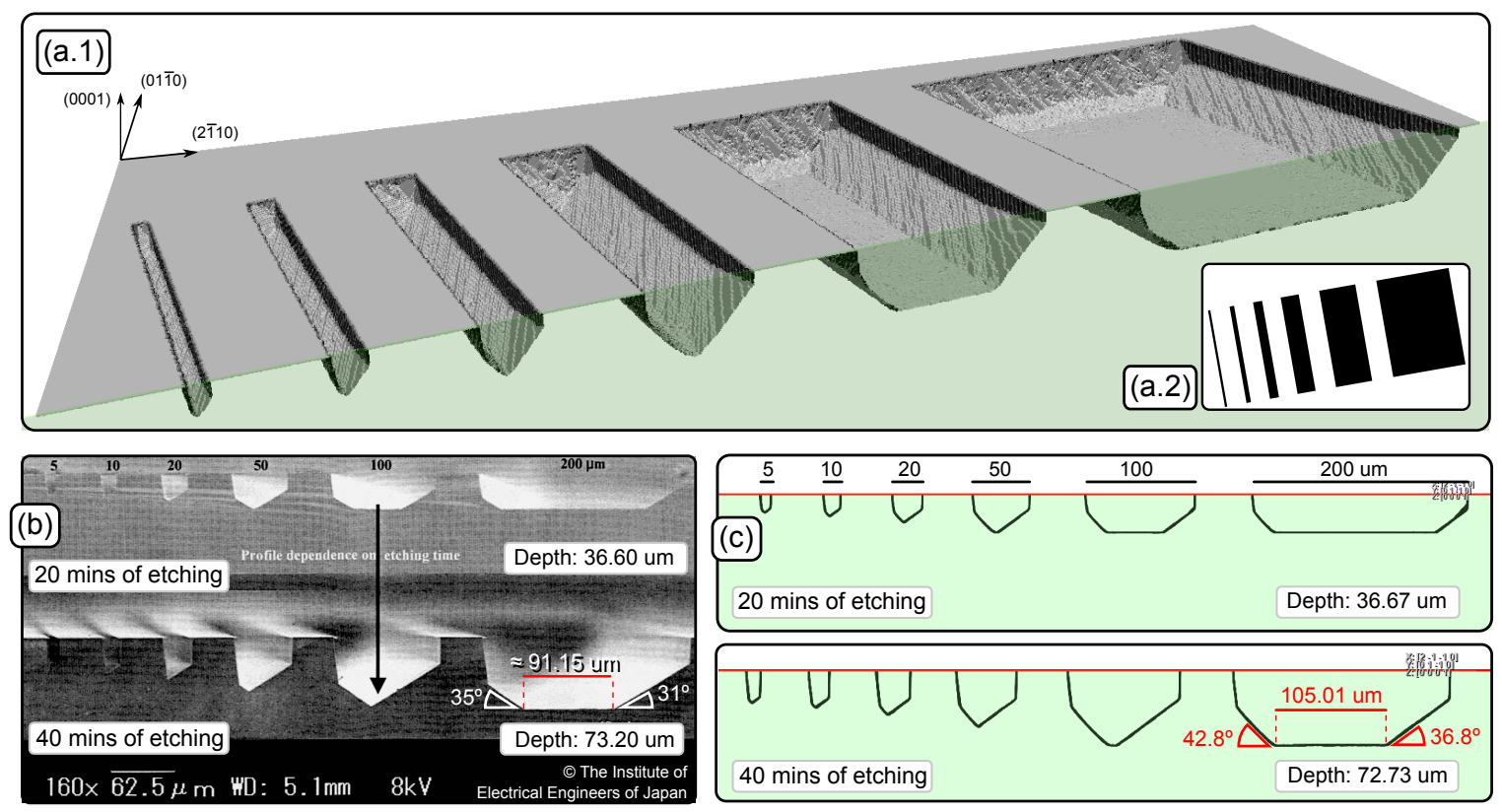

Figure 7. Etching of grooves in $\mathrm{NH}_{4} \mathrm{HF}_{2}$ at $85^{\circ} \mathrm{C}$ on a (0001) quartz substrate: (a) Simulator: 40 mins of etching, 3D view and applied mask. (b) Experiment: groove profiles after 20 and $40 \mathrm{~min}$ [15]. (c) Simulator: groove profiles after 20 and $40 \mathrm{~min}$.

(section 4.1), numerous simulations are performed in order to address the accuracy of the evolutionary CCA method.

Figure 7 compares the experimental and simulated results for the etching of a set of six grooves with a rotation angle of $10^{\circ}$ from the $\langle 01 \overline{1} 0\rangle$ direction. The substrate orientation is $\langle 0001\rangle$ and the mask is made of $\mathrm{Cr} / \mathrm{Au}$. The width of the grooves varies from 5 to $200 \mathrm{um}$. The etching process consists of two immersions of 20 minutes, taking a micrograph after each. Figures $7(\mathrm{a})$ and (c) show the results obtained by the CCA model while figure 7(b) shows the experimental results. From an experimental perspective, the angles of the developed sidewalls may vary from one wafer to another and even between equivalent regions of the same wafer. Keeping this in mind, the match between the experimental and simulated sidewall profiles for the different grooves in figure 7(b)-(c) is excellent in our opinion.

For a more quantitative comparison, we perform an extensive computational study that resembles the actual experiment presented in section 3.1 of Ref. [15]. The experiment consists in the etching of a set of 24 grooves, each rotated 5 degrees with respect to the previous one (figure 8(a)), covering a total range of $120^{\circ}$ (3-fold symmetry). For each groove, the sidewall profile is analyzed by measuring the angles $\alpha_{1}, \alpha_{2}$ and $\alpha_{3}$ as well as the etch rates $V_{1}, V_{2}$ and $V_{3}$ of the developed facets (figure $8(\mathrm{~b})$ ). The angles and rates have been used traditionally to characterize the quartz etch rate distribution, providing complete information about the etch profiles for differently oriented grooves.

Figure 9 shows a comparison of the sidewall angles and rates for the calibrated 


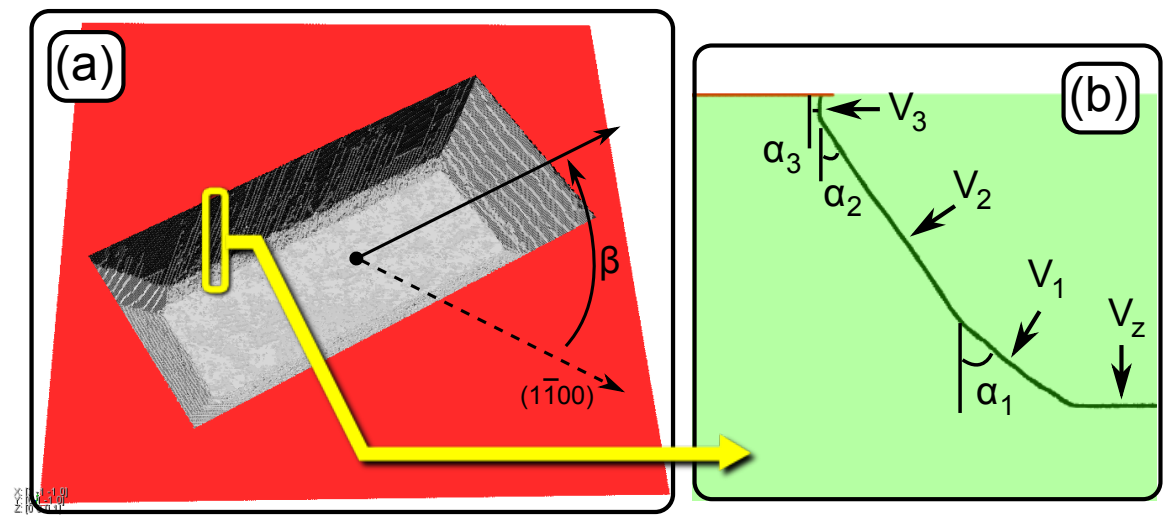

Figure 8. (a) Groove geometry for sidewall profile characterization. (b) Measured parameters: facet angles $\left(\alpha_{1}, \alpha_{2}\right.$ and $\left.\alpha_{3}\right)$ and facet etch rates $\left(V_{1}, V_{2}\right.$ and $\left.V_{3}\right)$.

CCA model and the experiment [15]. Although the overall match is excellent, some small differences can be pointed out, including: (i) $V_{1}$ and $V_{2}$ are slightly faster in the simulations (roughly 10\%), (ii) for grooves oriented in the range $30^{\circ} \leq \beta \leq 90^{\circ}$ the facets 2 and 3 are distinguished in the experiment as slightly different sidewalls (in orientation and etch rate) while they are merged into the same facet in the simulations, and (iii) for grooves oriented in the range $100^{\circ} \leq \beta \leq 115^{\circ}$ the angle $\alpha_{2}$ and etch rate $V_{2}$ show a discontinuity at $105^{\circ}$ in the experimental results while a smooth behavior is found in the simulations. Items (i) and (ii) are explained by small differences between the etch rate distributions used during the evolutionary calibration process and the actual experiment. The origin of item (iii) is unknown.

Finally, a set of tests has been performed in order to check the accuracy of the simulation for quartz-based, high-aspect-ratio MEMS. Used for creating tilt sensors [24], these structures make use of the slow etch rate of the $m$ plane and its vicinal orientations in order to obtain narrow structures with a large etch depth. The structures require long etch times and, thus, the prolonged competition between various slow planes that must be calibrated accurately. Figure 10 compares the simulated groove profiles and the experiment [15]. The mask opening is $5 \mu \mathrm{m}$ and each groove is rotated 5 degrees with respect to the previous one. The etch time is $40 \mathrm{~min}$. The CCA results are very close to the experiment for most groove orientations, demonstrating that the simulator maintains the global etch rate anisotropy of the experiment. The prolonged competition between slow planes makes the final structure very sensitive to small variations in the reactivity of the different planes.

\section{Conclusions}

A novel atomistic method for simulating anisotropic wet chemical etching of quartz is presented. The time evolution is obtained by using the Continuous Cellular Automaton (CCA) method. The crystallographic structure of quartz is implemented by using a 

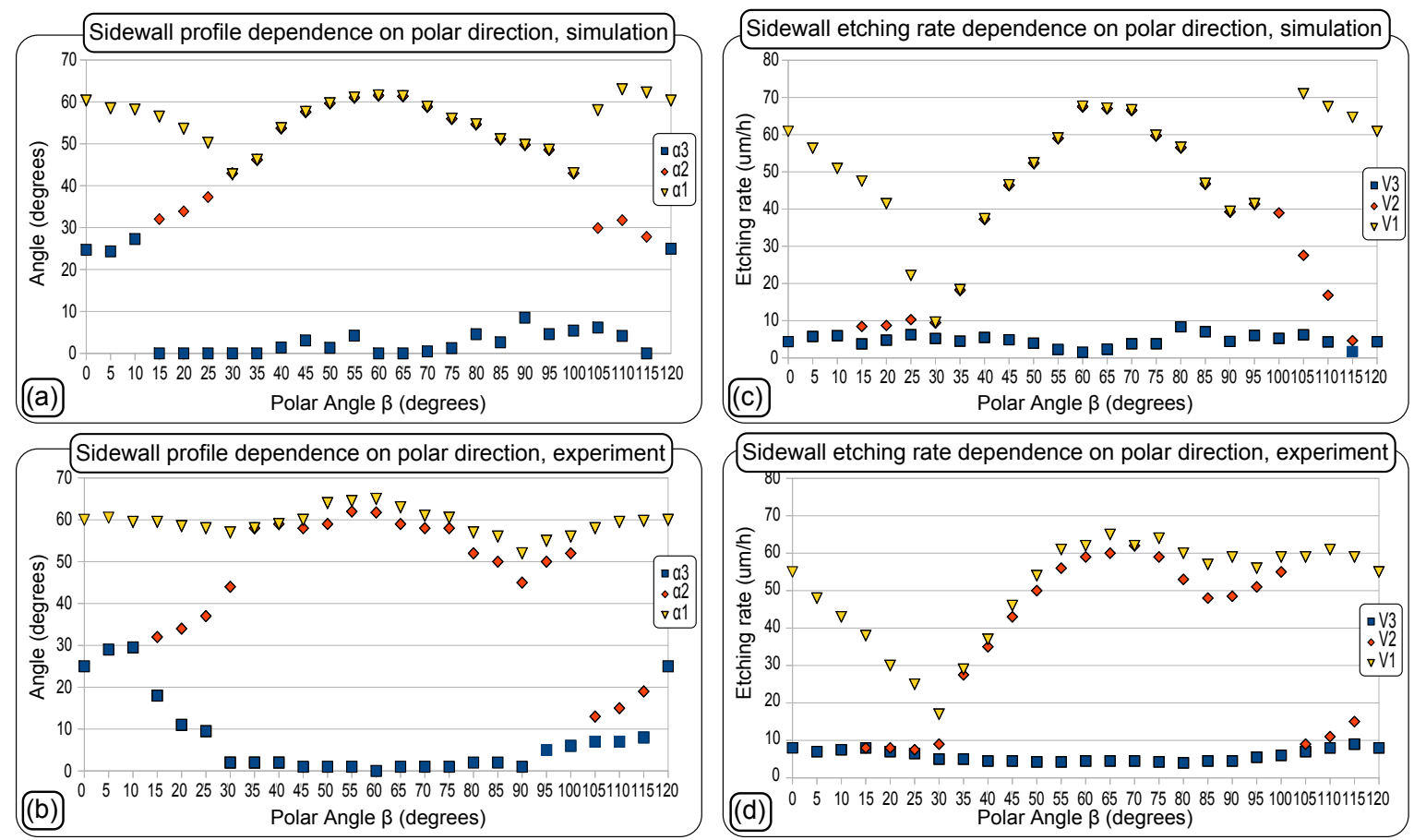

Figure 9. Simulated and experimental results for sidewall features $\left(\alpha_{1}, \alpha_{2}, \alpha_{3}, V_{1}\right.$, $V_{2}$ and $V_{3}$, cf. figure 8) vs groove rotation angle $(\beta)$. (a)-(b) Angle of the facets $\left(\alpha_{i}\right)$. (c)-(d) Etch rate of the facets $\left(V_{i}\right)$.

simplified unit cell that ignores the oxygen atoms and keeps the silicon atoms. As a result, we differentiate between two types of silicon nearest neighbors (black and white silicons) as well as between different nearest and next-nearest silicon neighbors, depending on their surface or bulk location. This gives rise to a novel classification of the surface atoms by using six indices and an Evolutionary Algorithm (EA) that is capable of assigning physically meaningful values to the corresponding atomistic removal rates. The resulting Evolutionary Continuous Cellular Automaton (ECCA) reproduces the correct macroscopic etch rate distribution for quartz hemispheres etched in saturated $\mathrm{NH}_{4} \mathrm{HF}_{2}$ at several temperatures and performs accurate, full 3D simulations of MEMS structures, as demonstrated by a quantitative comparison of numerous simulated and experimental trench profiles.

This new method constitutes a leap forward in comparison to the reduced number of existing quartz simulators, which so far have been primarily based on geometric methods of limited performance. Available as part of the IntelliEtch module [23], the new quartz etching simulator is expected to speed up the design flow of quartz MEMS based on bulk micromachining. Stepping away from silicon, the present paper demonstrates that the CCA method is suitable for modeling the anisotropic etching of other substrates, opening the door for a wide range of materials, such as GaAs, InP, copper or glass. 

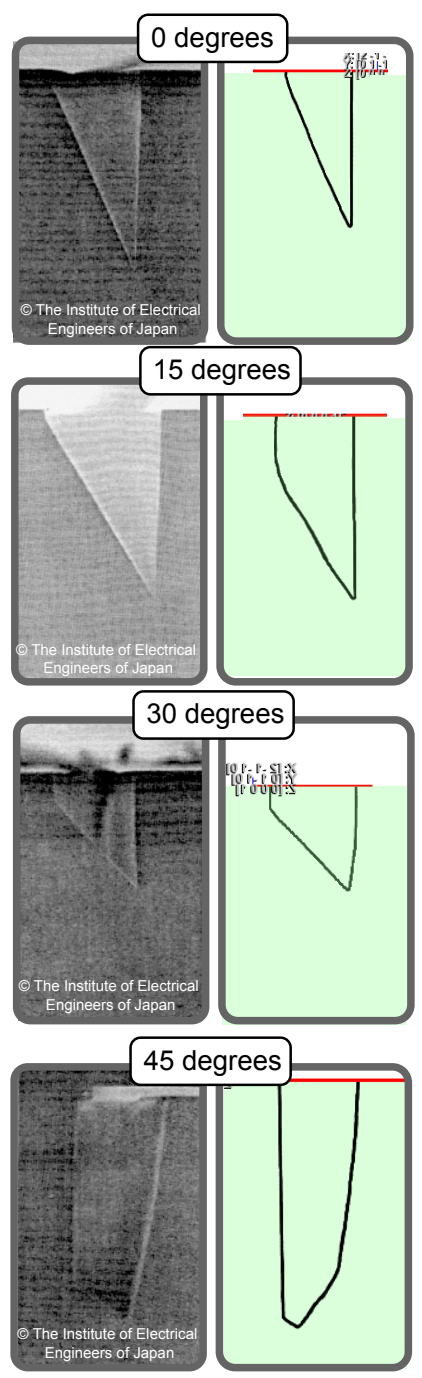
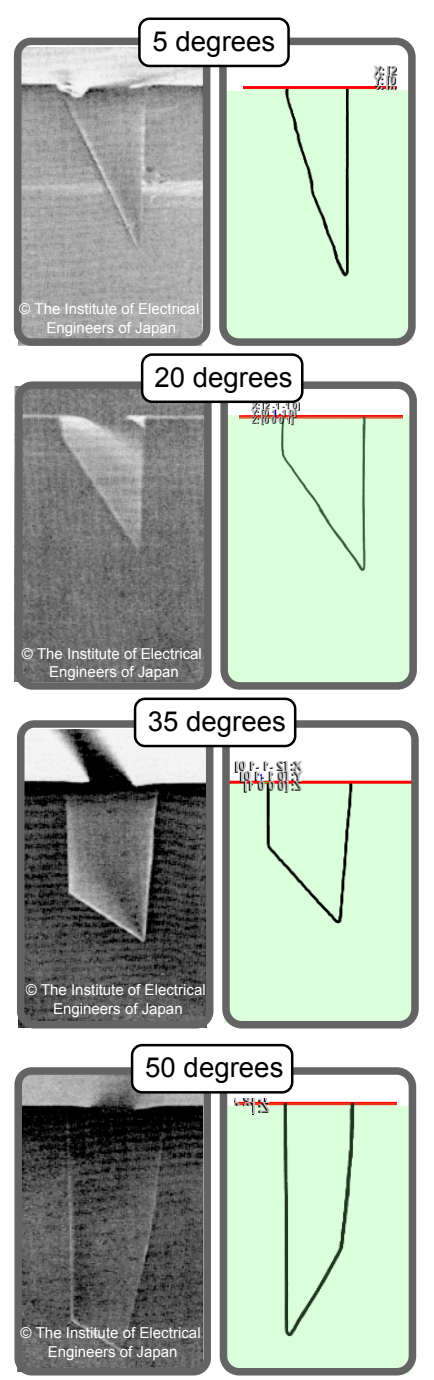

$20 \mathrm{um}$
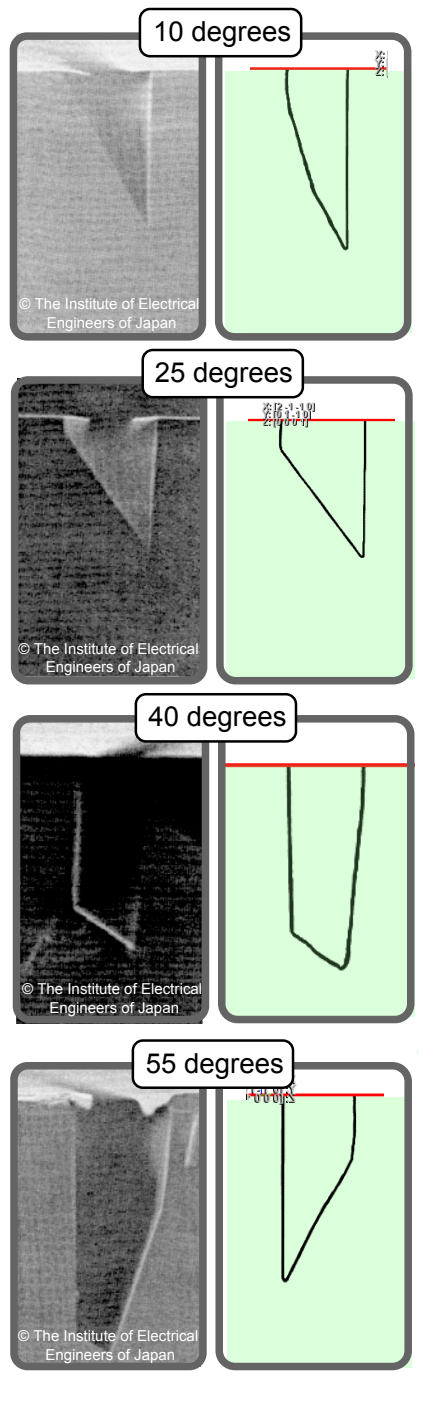

Figure 10. Sidewall profiles of high aspect ratio grooves for a rotation angle from 0 to 60 degrees. For each degree, left: experiment[15], right: CCA simulation.

\section{Acknowledgements}

We are grateful to D. Cheng and K. Sato (Nagoya University, Japan) for providing part of the experimental data. We acknowledge support by the Ramón y Cajal Fellowship Program by the Spanish Ministry of Science and Innovation, Spanish CICYT FIS201021216-C02-02 grant and the Professor Partnership Program by NVIDIA Corporation.

\section{References}

[1] M Lutz, A Partidge, P Gupta, N Bunchan, E Klaasen, J McDonald, and K Petersen. Mems oscillators for high volume commercial applications. In Transducers and Eurosensors '07 Conference Proceedings, pages 49-52. IEEE, 2007. 
[2] H Hida, M Shikida, K Fukuzawa, S Murakami, Ke Sato, K Asaumi, Y Iriye, and Ka Sato. Fabrication of a quartz tuning-fork probe with a sharp tip for afm systems. Sensors and Actuators A, 148:311-318, 2008.

[3] H Oh, G Kim, H Seo, Y Song, K Lee, and S S Yang. Fabrication of micro-lens array using quartz wet etching and polymer. Sensors and Actuators A, 164:161-167, 2010.

[4] Y Xing, M A Gosalvez, and K Sato. Step flow-based cellular automaton for the simulation of anisotropic etching of complex mems structures. New Journal of Physics, 9:436, 2007.

[5] Z Zhou, Q Huang, W Li, and W Deng. A cellular automaton-based simulator for silicon anisotropic etching processes considering high index planes. Journal of Micromechanics and Microengineering, 17:S38-S49, 2007.

[6] M A Gosalvez, Y Xing, and K Sato. Analytical solution of the continuous cellular automaton for anisotropic etching. Journal of Micromechanical Systems, 17(2):410-431, 2008.

[7] Z F Zhou, Q A Huang, and W H Li. Modeling and simulations of anisotropic etching of silicon in alkaline solutions with experimental verification. Journal of the Electrochemical Society, 156:F29-F37, 2009.

[8] M A Gosalvez, N Ferrando, Y Xing, P Pal, and K Sato. Simulating anisotropic etching of silicon in any etchant: evolutionary algorithm for the calibration of the continuous cellular automaton. Journal of Micromechanics and Microengineering, 21:065017, 2011.

[9] M Madani, D R Lankireddy, and T Nian-Feng. Gaseous sensors with area- and energy-efficient microhotplates through silica aerogel for heat insulation. In 2009 International Conference on Microelectronics (ICM), Conference Proceedings, pages 402-405. IEEE, 2009.

[10] B Tang, K Sato, H Tanaka, and M A Gosalvez. Fabrication of sharp tips with high aspect ratio by surfactant-modified wet etching for the afm probe. In 24th International Conference on Micro Electro Mechanical Systems (MEMS), Conference Proceedings, pages 328-331. IEEE, 2011.

[11] D Cheng, Ka Sato, M Shikida, A Ono, Ke Sato, K Asaumi, and Y Iriye. Characterization of orientation-dependent etching properties of quartz: Application to 3-d micromachining simulation system. Sensors and Materials, 17(4):179-186, 2005.

[12] P Rangsten, C HedLund, I V Katardjiev, and Y Bcklund. Etch rates of crystallographic planes in z-cut quartzexperiments and simulation. Journal of Micromechanics and Microengineering, 8:1-6, 1998.

[13] C R Tellier and T G Leblois. Micromachining of quartz plates: Determination of a database by combined stereographic analysis and 3-d simulation of etching shapes. IEEE Transactions on Ultrasonics, Ferroelectrics and Frequency Control, 47(5):1204-1216, 2000.

[14] P Rangsten, C HedLund, I V Katardjiev, and Y Bcklund. Anisotropic etching of z-cut quartz. Journal of Micromechanics and Microengineering, 3:65-73, 1993.

[15] J Liang, F Kohshaka, T Matsuo, and T Ueda. Wet etched high aspect ratio microstructures on quartz for mems applications. IEEJ Transactions on Sensors and Micromachines, 127:337-342, 2007.

[16] M A Gosalvez, Y Xing, K Sato, and R M Nieminen. Discrete and continuous cellular automata for the simulation of propagating surfaces. Sensors and Actuators A: Physical, 155(1):98-112, 2009.

[17] Z Zhu and C Liu. Micromachining process simulation using a continuous cellular automata method. Journal of Micromechanical Systems, 9(2):252-261, 2000.

[18] M A Gosalvez, Y Xing, K Sato, and R M Nieminen. Atomistic methods for the simulation of evolving surfaces. Journal of Micromechanics and Microengineering, 18:055029, 2008.

[19] N Ferrando, M A Gosalvez, J Cerda, R Gadea, and K Sato. Octree-based, gpu implementation of a continuous cellular automaton for the simulation of complex, evolving surfaces. Computer Physics Communications, 182(3):628-640, 2011.

[20] M Mitchell, P T Hraber, and J P Crutchfield. Revisiting the egde of chaos: Evolving cellular automata to perform computations. Complex Systems, 7:89-130, 1993.

[21] H Mhlenbein and D Schlierkamp-Voosen. Predictive models for the breeder genetic algorithm: I. 
continuous parameter optimization. Journal Evolutionary Computation, pages 25-49, 1993.

[22] K Sato, M Shikida, Y Matsushima, T Yamashiro, K Asaumi, Y Iriye, and M Yamamoto. Characterization of orientation-dependent etching properties of single-crystal silicon: effects of koh concentration. Sensors and Actuators A, 64:87-93, 1998.

[23] IntelliSense-Corp. Intellietch webpage. http://www.intellisense.com/modules/IntelliEtch. html, Aug. 2011.

[24] F Kohsaka, J Liang, T Matsuo, and T Ueda. High sensitive tilt sensor for quartz micromachining. IEEJ Transactions on Sensors and Micromachines, 127(10):431-436, 2007. 